\title{
Paediatric multisystem inflammatory syndrome temporally associated with SARS-CoV-2: a case report
}

Gilbert T Chua ${ }^{1 \dagger}$, MB, BS, FHKAM (Paediatrics), Joshua SC Wong ${ }^{2 \dagger}$, MB, BS, FHKAM (Paediatrics), Jaime Chung ${ }^{2}$, MB, BS, Ivan Lam², FHKCP, FHKAM (Paediatrics), Joyce Kwong ${ }^{3}$, FHKAM (Pathology), Kate Leung ${ }^{3}$, FRCPA, FHKAM (Pathology), CY Law 4 , PhD, FHKAM (Pathology), CW Lam 5 , PhD, FRCP, Janette Kwok', PhD, FRCPA, Patrick WK Chu', MPhil,

Elaine $\mathrm{YL} \mathrm{Au}^{7}$, FRCPA, FHKCPath, Crystal K Lam ${ }^{7}$, MB, BS, Daniel Mak'2, MRCPCH, FHKAM (Paediatrics),

NC Fong'2, FRCPCH, Daniel Leung', PhD (Candidate), Wilfred HS Wong', PhD, Marco HK Ho', MDM, FRCP,

Sabrina SL Tsao', MB, BS, FACC, Christina S Wong', MRCP, FHKAM (Medicine), Jason C Yam9 , MB, BS, FCOphthHK, Winnie WY Tso ${ }^{1}$, FHKAM (Paediatrics), Kelvin KW To ${ }^{10}$, MD, FRCPath, Paul KH Tam ${ }^{11,12}$, FRCS, FRCPCH,

Godfrey CF Chan', MD, FRCPCH, WH Leung', MB, BS, PhD, KY Yuen ${ }^{10}$, MD, FRCPath, Vas Novelli ${ }^{13,14}$, FRCP, FRCPCH, Nigel Klein ${ }^{13,14}$, PhD, Michael Levin ${ }^{15}$, PhD, FRCPCH, Elizabeth Whitaker ${ }^{16}$, MRCPCH, PhD, YL Lau', MD (Hon), FRCPCH,

Patrick Ip ${ }^{1}$, MPH, FHKAM (Paediatrics), Mike YW Kwan ${ }^{2}$, MRCPCH, MSc (Applied Epidemiology CUHK)

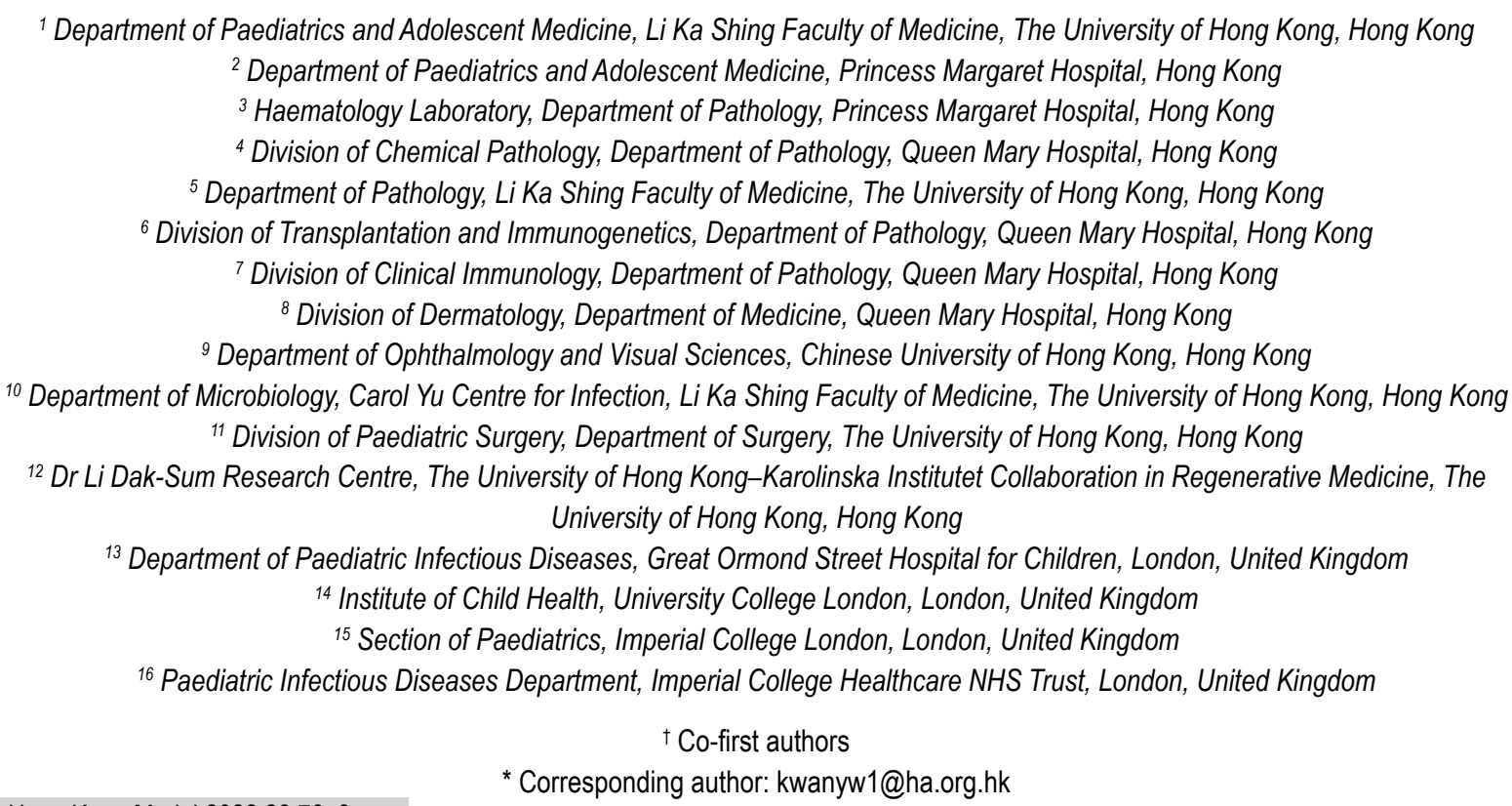

*Corresponding author: kwanyw1@ha.org.hk

Hong Kong Med J 2022;28:76-8

https://doi.org/10.12809/hkmj219689

\section{Case report}

A 10-year-old ethnic-Russian boy was confirmed to have severe acute respiratory syndrome coronavirus 2 (SARS-CoV-2) during the second wave of the coronavirus disease 2019 (COVID-19) outbreak in Hong Kong. ${ }^{1}$ He had a past medical history of coarctation of the aorta with corrective surgery performed at age 2 months. He returned from Russia on6 June 2020 and his first deep throat saliva specimen saved on arrival at Hong Kong International Airport tested negative for SARS-CoV-2. Four days later, he developed fever, malaise, and headache. On 13 June 2021, he was admitted to our Paediatric Infectious Disease Unit and a new deep throat saliva specimen was positive for SARS-CoV-2. He did not require oxygen during his hospital stay. He was discharged from the hospital after being tested positive for SARS-CoV-2 anti-nucleoprotein immunoglobulin G antibodies 17 days after admission. This complied with the discharge criteria set by the Department of Health, the Government of Hong Kong Special Administrative Region. ${ }^{1}$

On 16 July, 16 days after being discharged, he returned to our Paediatric Infectious Disease Unit with a 2-day history of high fever and right cervical tender lymphadenopathy. Repeat nasal pharyngeal swab for SARS-CoV-2 polymerase chain reaction was negative. He was presumed to have bacterial lymphadenitis and was prescribed intravenous antibiotics but symptoms progressed. Ultrasound of the neck showed evidence of lymphadenitis but no signs of abscess formation. His fever and lymphadenitis persisted for 5 days and he also developed bilateral non-purulent conjunctivitis with peri-limbic sparing, erythematous and cracked lips, strawberry tongue and blanchable erythema over the trunk (Fig). Serial blood tests showed mild thrombocytopenia (trough $110 \times 10^{9} / \mathrm{L}$ ), and raised 


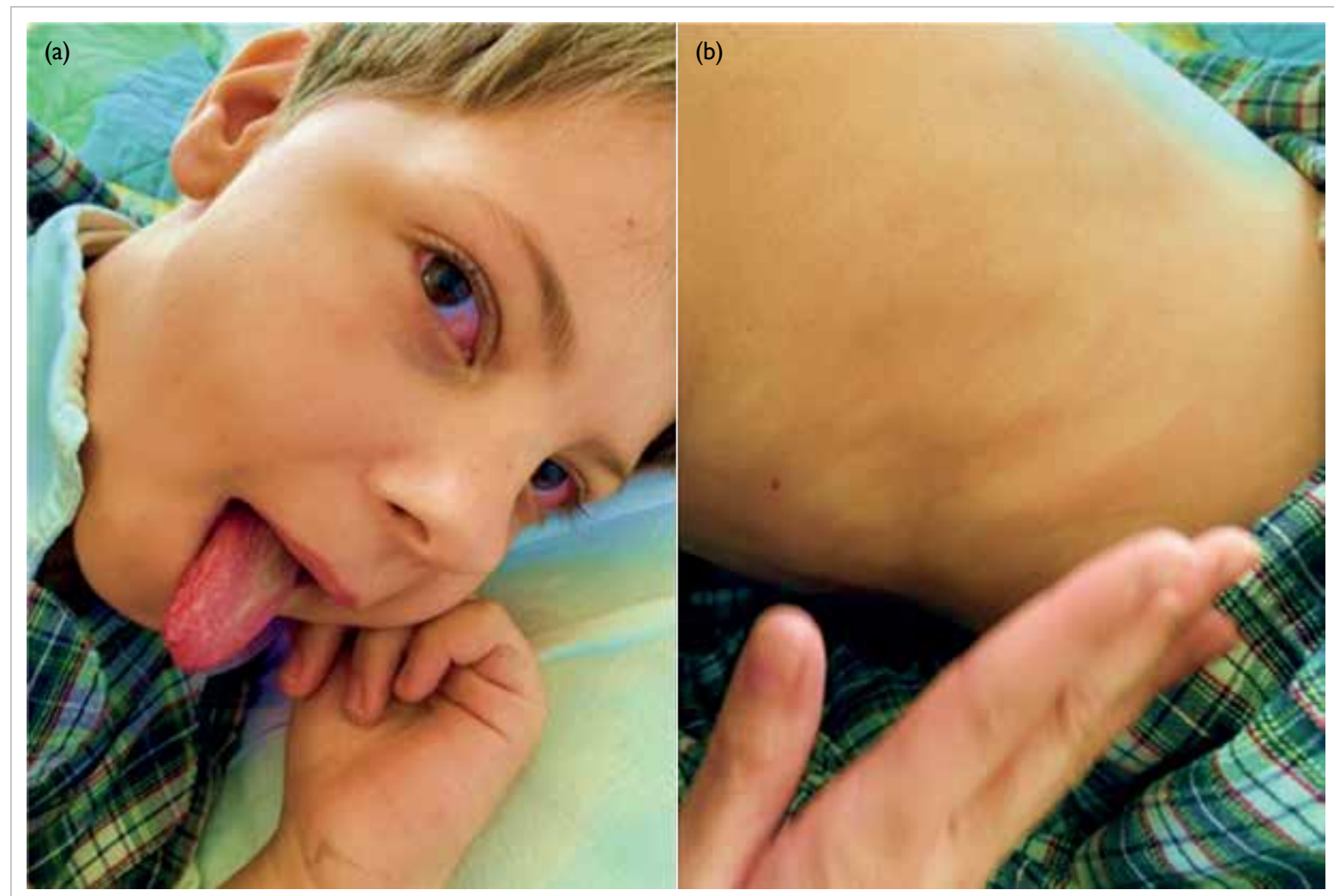

FIG. Clinical photographs showing (a) bilateral conjunctival injection with peri-limbic sparing and strawberry tongue, and (b) blanchable erythema over trunk. (Written consent for publication obtained from the patient's parents)

erythrocyte sedimentation rate (peak $60 \mathrm{~mm} / \mathrm{Hr}$ ), C-reactive protein (peak $102 \mathrm{mg} / \mathrm{L}$; range, <5.0), lactate dehydrogenase (270 U/L; range, <270), ferritin (1568 pmol/L; range, 31-279), highly sensitive troponin I (peak $643 \mathrm{ng} / \mathrm{L}$; range, <21), N-terminal prohormone of brain natriuretic peptide (peak $3213 \mathrm{ng} / \mathrm{L}$; range, <112), and interleukin-6 (IL-6) (peak $480.9 \mathrm{pg} / \mathrm{mL}$; range, <4). Electrocardiogram and echocardiogram were unremarkable. His clinical presentation was compatible with Kawasaki-like disease. Since he had been infected with COVID-19 approximately 4 weeks previously, he was suspected to have PIMS-TS (paediatric multisystem inflammatory syndrome temporally associated with SARS-CoV-2). ${ }^{2}$ Other differential diagnoses were excluded but included streptococcal and staphylococcal infection, Epstein-Barr virus infection and infection-related myocarditis. Owing to the rarity of PIMS-TS in East Asia, the clinical team discussed the case with experts from the United Kingdom who concurred with the diagnosis. He was treated with two doses of intravenous immunoglobulin (IVIG) at $2 \mathrm{~g} / \mathrm{kg} / \mathrm{dose}$ as his fever resurged 1 day after the first dose. Fever and other symptoms subsequently subsided after the second dose of IVIG, and serial echocardiograms did not reveal any coronary lesions. Whole exome sequencing performed to look for the possibility of an underlying monogenic immune dysregulation syndrome because of the rarity of this condition was unremarkable.

\section{Discussion}

Paediatric multisystem inflammatory syndrome temporally associated with SARS-CoV-2 is one of the most severe complications of COVID-19 infection in children. It was initially described in several case series in Europe and North America among children who presented with Kawasaki-like illness and were confirmed or known to have been in contact with another SARS-CoV-2-infected individual. ${ }^{2,3}$ Kawasaki disease (KD) is most prevalent among East Asians but rare in other parts of the world. ${ }^{3}$ On the contrary, paediatric cross-sectional clinical studies from East Asia have reported that PIMS-TS is rare among East Asians. ${ }^{1}$ We present the first, and so far, the only case of PIMS-TS in China. The case was an ethnic Russian boy who showed features of KD approximately 4 weeks after confirmation of SARS-CoV-2 infection. There is no consensus on the treatment regimen at present. In our patient, IVIG alone, instead of steroid or immunomodulators, was effective in treating the condition.

The pathophysiology of PIMS-TS remains uncertain. Studies have shown significant clinical and laboratory differences between PIMS-TS 
and KD, despite some similarities in clinical be sought.

presentation. Patients with PIMS-TS are generally

older than those with KD (median age, 8.3-9 years vs 2.7 years). ${ }^{2,3}$ They also have a higher white blood cell and neutrophil count and $\mathrm{C}$-reactive protein, and a greater degree of lymphopenia and anaemia and tendency to develop thrombocytopenia in contrast to thrombocytosis in KD. In addition, fibrinogen and troponin levels are more elevated in PIMS-TS. ${ }^{2}$ These factors are associated with an increased risk of intensive care admission among children with PIMS-TS. ${ }^{2}$ These findings imply that PIMS-TS is a different entity to $K D$, with a greater degree of inflammation and myocardial injury. Studies have shown that certain cytokines, such as IL-6, appear to be particularly elevated in patients with PIMS-TS and may be involved in myocardial depression. ${ }^{2}$ Studies have also suggested that life-threatening COVID-19 pneumonia may be associated with monogenic inborn errors of immunity related to type 1 interferonopathies or type 1 interferon neutralising antibodies. ${ }^{4}$ Certain human leukocyte antigens, which are prevalent in East Asians but not Caucasians, have been associated with KD. ${ }^{4}$ However, no genes have been identified to cause PIMS-TS. Future studies will continue to explore the genetic factors related to PIMS-TS and the possible associated leukocyte antigen that explains the ethnic differences in PIMS-TS prevalence.

The treatment for PIMS-TS is similar to that for KD. A recent observational study demonstrated that patients who received IVIG and methylprednisolone together were less likely to require second-line biological agents, and were at lower risk of secondary acute left ventricular dysfunction and need for haemodynamic support with a shorter length of stay in the intensive care unit. ${ }^{5}$ Interleukin-1 and IL-6 receptor monoclonal antibodies have been used as second-line biological agents and have been shown to achieve remission when first-line therapies fail. ${ }^{2,5}$ Short-term outcomes of PIMS-TS are generally good. Immediate cardiac complications include coronary abnormalities, transient valvular regurgitation and myocardial dysfunction. ${ }^{2}$ The majority of patients recover without sequelae, but mortality has been reported. ${ }^{2}$ Data on the long-term outcomes of PIMS-TS are lacking.

The PIMS-TS remains a rare disease among East Asian patients. ${ }^{1}$ Nevertheless, frontline paediatricians in East Asia should remain vigilant when looking after ethnic non-East Asian children with COVID-19 infection in case they develop PIMS-TS after their initial recovery. Paediatricians should advise parents about the symptoms and signs of PIMS-TS so that timely medical consultation can

\section{Author contributions}

Concept or design: GT Chua, JSC Wong, P Ip, MYW Kwan. Acquisition of data: J Chung, I Lam, J Kwong, K Leung, CY Law, CW Lam, J Kwok, PWK Chu, EYL Au, CK Lam, MYW Kwan. Analysis or interpretation of data: D Mak, NC Fong, D Leung, WHS Wong, MHK Ho, SSL Tsao, CS Wong, JC Yam, WWY Tso, KKW To, PKH Tam, GCF Chan, WH Leung, KY Yuen, V Novelli, N Klein, M Levin, E Whitaker, YL Lau.

Drafting of the manuscript: GT Chua, JSC Wong, I Lam, J Chung.

Critical revision of the manuscript for important intellectual content: All authors.

All authors had full access to the data, contributed to the study, approved the final version for publication, and take responsibility for its accuracy and integrity.

\section{Conflicts of interest}

As an editor of the journal, JC Yam was not involved in the peer review process. Other authors have disclosed no conflicts of interest.

\section{Funding/support}

This work was supported by the Collaborative Research Fund (CRF) 2020/21 and One-off CRF Coronavirus and Novel Infectious Diseases Research Exercises (Ref: C7149-20G). The funding source was not involved in the study design, collection, analysis or interpretation of data; in the writing of the manuscript; or in the decision to submit the manuscript for publication.

\section{Ethics approval}

The patient was treated in accordance with the Declaration of Helsinki, and the parents of the patient provided informed consent for the treatment and procedures.

\section{References}

1. Chua GT, Wong JS, Lam I, et al. Clinical characteristics and transmission of COVID-19 in children and youths during 3 waves of outbreaks in Hong Kong. JAMA Netw Open 2021;4:e218824.

2. Whittaker E, Bamford A, Kenny J, et al. Clinical characteristics of 58 children with a pediatric inflammatory multisystem syndrome temporally associated with SARS-CoV-2. JAMA 2020;324:259-69.

3. To KK, Chua GT, Kwok KL, et al. False-positive SARS-CoV-2 serology in 3 children with Kawasaki disease. Diagn Microbiol Infect Dis 2020;98:115141.

4. Sancho-Shimizu V, Brodin P, Cobat A, et al. SARS-CoV-2-r elated MIS-C: A key to the viral and genetic causes of Kawasaki disease? J Exp Med 2021;218:e20210446.

5. Ouldali N, Toubiana J, Antona D, et al. Association of intravenous immunoglobulins plus methylprednisolone vs immunoglobulins alone with course of fever in multisystem inflammatory syndrome in children. JAMA 2021;325:85564. 